\title{
Economic considerations in the treatment of invasive aspergillosis: a review of voriconazole pharmacoeconomic studies
}

This article was published in the following Dove Press journal:

ClinicoEconomics and Outcomes Research

10 August 2009

Number of times this article has been viewed

\author{
Kem P Krueger \\ A Christie Nelson \\ School of Pharmacy, University \\ of Wyoming, Laramie, WY, USA
}

Correspondence: Kem P Krueger 1000 E University Avenue, Dept 3375, Laramie, WY 8207I, USA

Tel + I 307-766-6532

Fax + I 307-766-2953

Email kpkrueger@uwyo.edu

\begin{abstract}
Invasive aspergillosis is a life-threatening fungal infection predominately affecting immunocompromised individuals. The incidence of inpatient-treated aspergillosis cases in the US is estimated to be between 3.02 and 3.80 per 10,000 hospitalized patients. The estimated difference in hospital costs of patients with an aspergillosis infection is US $\$ 36,867$ to US\$59,356 higher than those of patients without the infection. Voriconazole is a synthetic, broad spectrum triazole antifungal agent, with FDA-approved indications for the treatment of invasive aspergillosis, esophageal candidiasis, candidemia in nonneutropenic patients, invasive candidiasis, and infections due to Scedosporium apiospermum and Fusarium species in patients refractory to or intolerant of other therapy. Eight cost-effectiveness analyses, one cost-minimization analysis, and one cost analysis were identified from a Medline search. The 10 pharmacoeconomic analyses were conducted in six different countries comparing voriconazole to conventional amphotericin B, liposomal amphotericin B, itraconazole, and caspofungin. All the cost-effectiveness and cost-minimization analyses identified voriconazole as the most cost-effective therapy. The cost analysis demonstrated voriconazole cost-savings. While the acquisition costs of voriconazole are higher than those of conventional amphotericin $\mathrm{B}$, the toxicity profile and rate of treatment success associated with voriconazole result in lower total treatment costs per successfully treated patient.
\end{abstract}

Keywords: voriconazole, antifungal agents, invasive aspergillosis, pharmacoeconomics

\section{Overview and management of invasive aspergillosis}

Invasive aspergillosis is a life-threatening fungal infection predominately affecting immunocompromised individuals. The infection is most commonly caused by Aspergillus fumigatus, with Aspergillus flavus, Aspergillus niger, and Aspergillus terreus less often implicated. ${ }^{1}$ Profound and prolonged neutropenia is the most common risk factor associated with development of disease. As such, hematopoietic stem cell transplant (HSCT) recipients and patients receiving aggressive chemotherapy for hematologic cancers are at particular risk. ${ }^{2,3}$

Invasive aspergillosis most frequently begins in the lungs after inhalation of Aspergillus conidia. Initial, nonspecific manifestations include fever, cough, and dyspnea. ${ }^{4}$ Without treatment, invasive pulmonary aspergillosis is almost always fatal. ${ }^{1,4,5}$

Definitive diagnosis of invasive aspergillosis requires culture confirmation of Aspergillus species after biopsy of a sterile specimen. However, culture results lack sensitivity, may take as long as 4 weeks, and be confounded by contamination., ${ }^{1,4}$ Prompt treatment is essential to improve survival. In the high-risk host, treatment is generally initiated based on signs and symptoms consistent with the disease. These may 
include fever despite the use of broad-spectrum antimicrobial agents, pulmonary infiltrates on CT scan (particularly with the presence of the halo or air-crescent sign), and/or detection of galactomannan antigen in the serum. ${ }^{1}$

Several antifungal agents are indicated for the treatment of invasive aspergillosis, including amphotericin B and its lipid formulations, itraconazole, voriconazole and caspofungin. Voriconazole, however, is the drug of choice for the primary treatment of invasive aspergillosis. ${ }^{1}$ Voriconazole is initiated with a loading dose of $6 \mathrm{mg} / \mathrm{kg}$ intravenously (iv) every 12 hours for two doses and followed by $4 \mathrm{mg} / \mathrm{kg}$ every 12 hours. Parenteral administration is recommended in seriously ill patients, but clinically stable patients may be converted to oral tablets (with weight-based dosing rounded up to convenient pill sizes). A minimum of 6 to 12 weeks of therapy is required to adequately treat invasive pulmonary aspergillosis. Longer durations are necessary when prolonged periods of immune suppression are present and in cases where infected lesions are slow to resolve. ${ }^{1}$ Voriconazole levels have been correlated with antifungal efficacy and toxicity in several small studies. ${ }^{6,7}$ Though not yet routinely advocated, therapeutic drug monitoring is reasonable when drug toxicity or refractory fungal disease is suspected. ${ }^{4}$

The incidence of inpatient-treated aspergillosis cases in the US in the late 1990s was between 3.02 and 3.80 per 10,000 hospital patients, representing over 10,000 aspergillosis-related discharges from community hospitals. ${ }^{8,9}$ The total US community hospital-related cost of aspergillosis in 1996 was estimated to be US\$633.1 million. ${ }^{8}$ The estimated difference in hospital costs of patients with aspergillosis in the late 1990s were US\$36,867 to US\$59,356 higher than those of patients without the infection. ${ }^{8,9}$ Using the US Bureau of Labor Statistics CPI Inflation Calculator, the equivalent 2009 amounts are US\$48,110 to US\$80,468. ${ }^{10}$ Compared to patients with other invasive fungal infections such as candidiasis, histoplasmosis, and cryptococcosis, those with aspergillosis had the highest per-patient treatment costs (US\$37,921/patient for inpatient care and US\$34,871/patient for outpatient follow-up care). ${ }^{9}$ When adjusted to 2009 dollars the total cost to treat a patient with aspergillosis is US\$94,991 which is consistent with 2003 estimates provided by Tong and colleagues. ${ }^{11}$

Although voriconazole is considered first-line therapy for the primary treatment of invasive aspergillosis, it does not have the lowest acquisition cost of the FDA-approved treatments of invasive aspergillosis. Therefore the purpose of this article is to review the pharmacoeconomic studies that included voriconazole for the treatment of invasive aspergillosis. An overview of voriconazole is provided as background information.

\section{Overview of voriconazole}

Voriconazole is a synthetic, broad spectrum triazole antifungal agent that inhibits fungal cytochrome p450-dependent enzyme lanosterol $14-\alpha$-demethylase, ultimately decreasing the biosynthesis of ergosterol, an essential component of the fungal cell membrane. Voriconazole is FDA-approved for the treatment of invasive aspergillosis, esophageal candidiasis, candidemia in nonneutropenic patients, invasive candidiasis and infections due to Scedosporium apiospermum and Fusarium species in patients refractory to or intolerant of other therapy. ${ }^{12}$

Voriconazole is available as both oral and parenteral formulations. The oral tablet has a bioavailability exceeding 90\%, which permits switching between formulations when clinically appropriate. The kinetics of voriconazole are nonlinear, dose-dependent, and exhibit a high degree of inter-patient variability. It is metabolized by the P450 (CYP) enzymes CYP2C19, CYP2C9, and CYP3A4 to inactive metabolites. Its metabolism is saturable; increasing doses of voriconazole result in disproportional increases in drug exposure. ${ }^{13}$

Voriconazole is generally well tolerated. The most commonly reported adverse reactions, occurring in at least 5\% of patients, include visual disturbances, elevated hepatic enzymes, fever, rash, nausea and vomiting. ${ }^{12,14,15}$ Visual and auditory hallucinations have also been occasionally reported in patients receiving voriconazole. ${ }^{12,15}$ Plasma voriconazole levels exceeding $5.5 \mu \mathrm{g} / \mathrm{mL}$ have been linked to higher incidences of neurologic events, visual disturbance and hepatotoxicity. ${ }^{6,7}$

Voriconazole has numerous drug-drug interactions, which may limit its usefulness in certain populations. Voriconazole is both a substrate and inhibitor of P450 (CYP) enzymes CYP2C19, CYP2C9, and CYP3A4. ${ }^{12,14,15}$

Voriconazole is available for IV use as a $200 \mathrm{mg}$ powder for reconstitution and for oral use as $200 \mathrm{mg}$ and $50 \mathrm{mg}$ tablets and a $200 \mathrm{mg} / 5 \mathrm{~mL}$ powder for suspension. ${ }^{12}$ Average wholesale prices are US\$121.63 per $200 \mathrm{mg}$ iv vial, US\$10.33 per $50 \mathrm{mg}$ tablet, and US\$41.33 per $200 \mathrm{mg}$ tablet. ${ }^{16}$

\section{Clinical outcomes}

Historically, invasive aspergillosis-associated mortality rates have ranged from $60 \%$ to $90 \%$, with central nervous system or disseminated infection or disease in HSCT patients 
conferring graver prognoses. ${ }^{17-20}$ Changes in transplantation practices, diagnostic procedures, and the extensive use of voriconazole have been credited with significant reductions in mortality rates. ${ }^{17,21}$

In 2002, Herbrecht and colleagues published the pivotal Global Comparative Aspergillosis (GCA) study sponsored by Pfizer. ${ }^{22}$ The investigators compared the efficacy and safety of voriconazole and conventional amphotericin $\mathrm{B}$ desoxycholate (CAB) for the primary treatment of invasive aspergillosis in immunocompromised patients. This prospective, randomized trial involved 277 patients $(n=144$, voriconazole; $n=133, \mathrm{CAB}$ ) aged 12 years or greater with definite or probable aspergillosis. Most had an underlying diagnosis of allogeneic HSCT or hematologic cancer and nearly half were neutropenic. The most common site of infection was the lungs. ${ }^{22}$

Voriconazole was dosed at $6 \mathrm{mg} / \mathrm{kg}$ iv every 12 hours $\times 2$ doses, then $4 \mathrm{mg} / \mathrm{kg}$ iv twice daily for at least 7 days, at which time it could be switched to oral administration of $200 \mathrm{mg}$ twice daily. CAB was dosed at 1.0 to $1.5 \mathrm{mg} / \mathrm{kg}$ iv daily. Other licensed therapy was permitted in the case of therapeutic failure or drug intolerance, which occurred in 52 patients in the voriconazole and 107 patients in the $\mathrm{CAB}$ groups. The planned duration of therapy was 12 weeks. ${ }^{22}$

At week 12, voriconazole was associated with significantly improved response rates and survival and fewer adverse reactions than CAB. Successful response, defined as clinical improvement or resolution and at least $50 \%$ improvement of radiologic findings, was achieved by $52.8 \%$ of patients receiving voriconazole and $31.6 \%$ of patients receiving $\mathrm{CAB}$ (absolute difference 21.2\%; $95 \%$ CI $10.4 \%$ to $32.9 \%$ ). The survival rate was $70.8 \%$ in the voriconazole group versus $57.9 \%$ in the $\mathrm{CAB}$ group (HR 0.59 ; 95\% CI 0.40 to 0.88). Renal impairment and infusion-related fevers and chills were more common in patients receiving $\mathrm{CAB}$ while voriconazole-treated patients were more likely to experience visual disturbances and dermatologic reactions. ${ }^{22}$

Based on the results of this trial, voriconazole replaced amphotericin B as first-line therapy for the treatment of invasive aspergillosis. A recent observational study using data compiled by the multicenter Prospective Antifungal Therapy (PATH) Alliance registry demonstrated mortality rates consistent with those reported by the Herbrecht trial. ${ }^{21}$ In this study, the epidemiology and outcomes of 144 HSCT patients with invasive aspergillosis were examined. Eighty-five percent of these patients received treatment with voriconazole, $47 \%$ in combination with at least one other antifungal agent. Six- and 12-week mortality rates were $21.5 \%$ and $35.5 \%$, respectively. ${ }^{21}$

\section{Economic outcomes}

A Medline search using the terms "cost analysis" or "pharmacoeconomic analysis" plus "aspergillosis" plus "voriconazole" was serially repeated, substituting voriconazole with caspofungin, amphotericin $\mathrm{B}$, and itraconazole. Studies were limited to those published in the English language that compared both the costs and outcomes of invasive aspergillosis therapy and included a voriconazole arm. Ten publications met the inclusion criteria. These articles are summarized in Tables 1 to 5. These ten studies are compared globally by the type of analysis, methods used to estimate the cost and outcomes, results, transparency, and other methodological issues.

The 10 pharmacoeconomic analyses were conducted in six different countries as indicated in Table 1. Voriconazole was compared to $\mathrm{CAB},{ }^{23-30}$ liposomal amphotericin $\mathrm{B}$ (L-AMB), ${ }^{31}$ itraconazole, ${ }^{24}$ and caspofungin. ${ }^{32}$ Generally speaking all of the studies used the appropriate procedures for conducting pharmacoeconomic analyses with regard to their research question, modeling procedures, data sources, resource valuation, transparency, discounting, use of sensitivity analyses, and use of incremental analyses. ${ }^{33}$ Any exceptions to these standards are noted in this section. Overall, the 10 studies had well-defined research questions, and they were transparent indicating that the data sources and methods for calculating expected costs and outcomes were obvious.

Eight of the studies were cost-effectiveness analyses (CEA), ${ }^{23-29,31}$ one was a cost-minimization analysis (CMA), ${ }^{32}$ and one was a cost analysis (CA). ${ }^{30}$ No cost-utility analyses of voriconazole were identified, which is not surprising given the limited treatment period and the overall poor health condition of people with invasive aspergillosis.

Eight of the voriconazole pharmacoeconomic studies used a decision analytic model such as a decision tree or Markov Model to estimate the expected costs and outcomes of therapy. ${ }^{23-29,31}$ One of the studies calculated the costs and outcomes from actual clinical observations and used a decision tree to calculate the weighted average cost and outcomes. $^{23}$

The three outcomes reported in the studies were successful response at 12 weeks, survival at 12 weeks, and mean survival time. Tables 2, 3, and 4 present the results according to each of these outcomes, respectively. Since the studies were conducted in different countries with different 


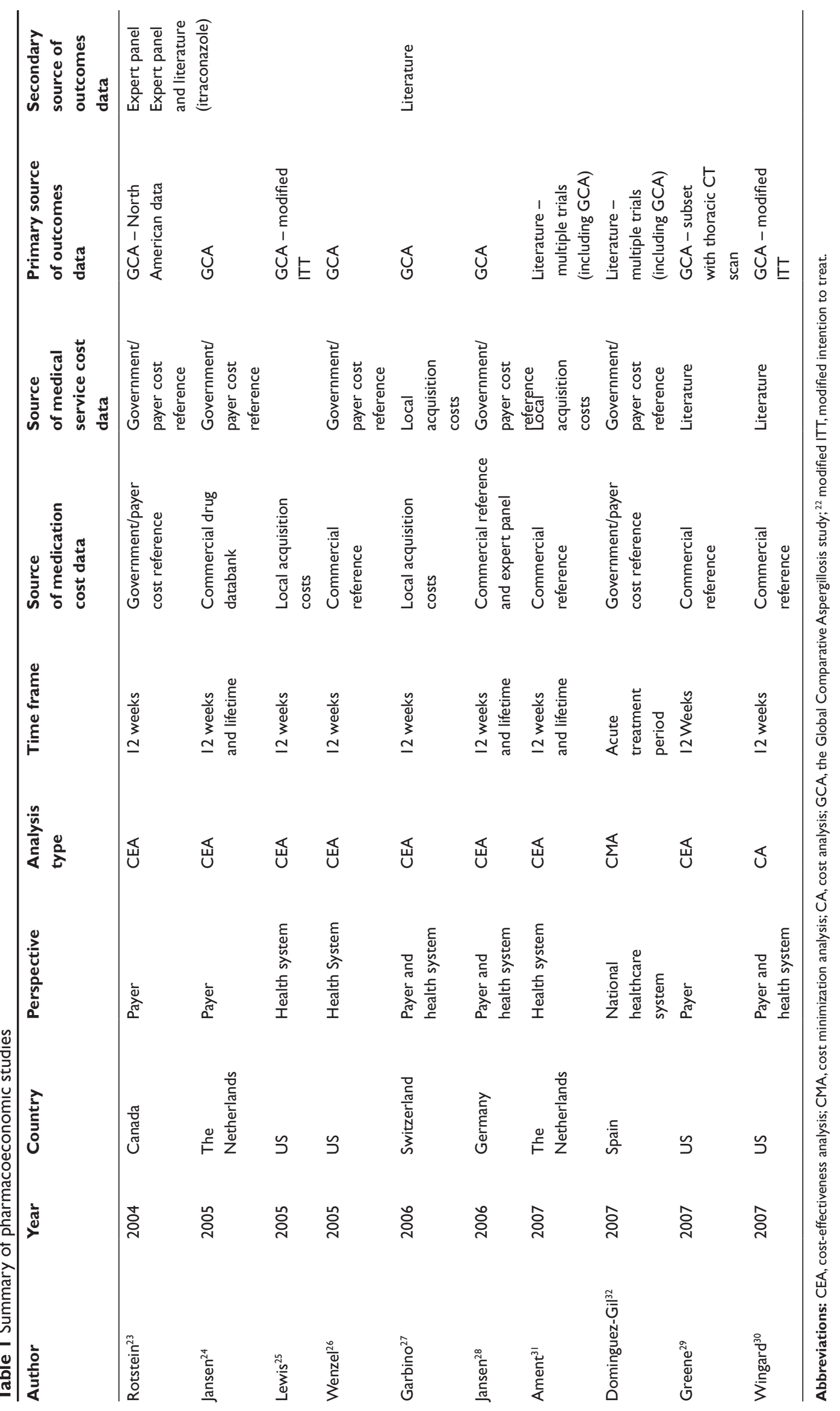




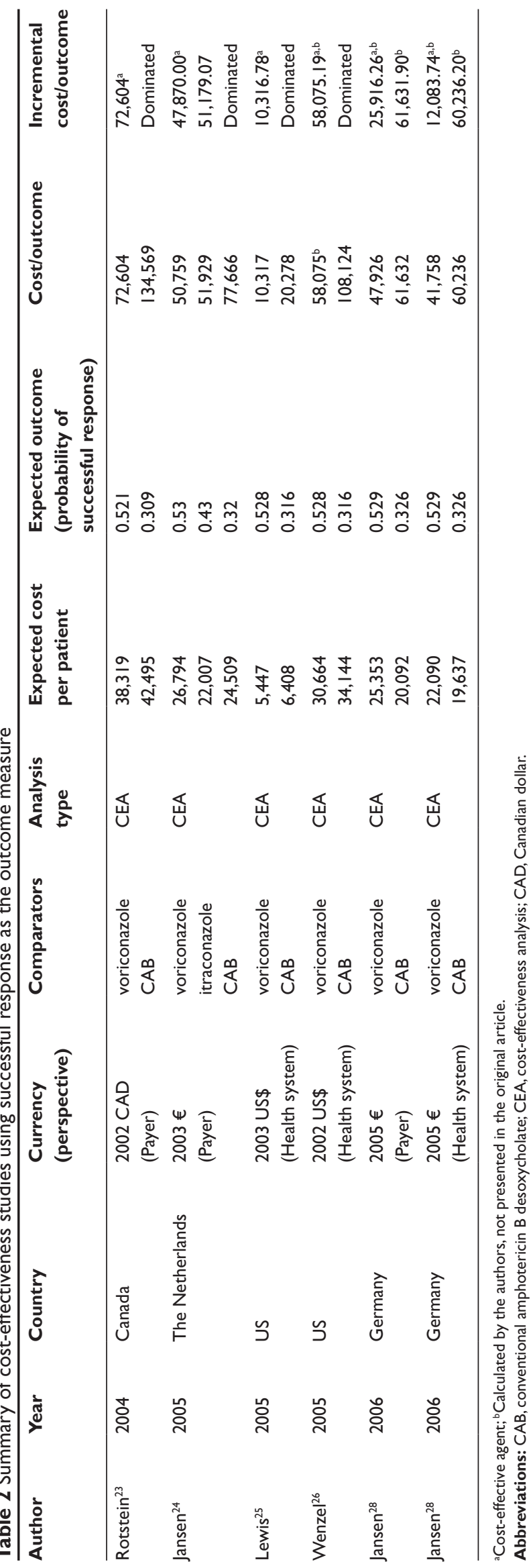

healthcare systems and different currencies, no attempt was made to combine the results. All cost-effectiveness analyses or cost-minimization analyses identified voriconazole treatment arms as the most cost-effective. This is not unexpected given the number of studies that used the results from GCA study as the primary source for outcomes data. However, the analyses that used multiple studies as the primary source for outcomes data reported similar results..$^{31,32}$

None of the studies discounted costs or outcomes. This is appropriate for all of the studies that used the first two outcome measures since the study periods were less than 12 months. Three studies reported mean survival times. Given that some of the costs and outcomes in these studies occurred outside the 12-month window, they should have been discounted. Most likely this is not a major issue given that the average life expectancy of the people included in these analyses ranged from less than 12 months to 3 years. ${ }^{24,28,31}$ These three studies are described in greater detail below.

Jansen and colleagues used the results of the GCA study ${ }^{22}$ as the basis of their decision tree which estimated 12 week cost and outcomes in a Dutch population. ${ }^{24}$ In the model, patients were treated initially with voriconazole, $\mathrm{CAB}$, or itraconazole and were switched to other licensed antifungal agents in the case of severe toxicity or treatment failure. Since itraconazole was not included in the GCA study, they used other literature and expert opinion to identify resource use and probabilities of response and toxicities in the itraconazole arm. A Markov Model was then used to simulate the lifetime costs and outcomes of voriconazole, itraconazole, and amphotericin B as first-line treatments for invasive aspergillosis. Patients treated successfully at the end of 12 weeks remained in the "success" state until they died (an assumption of no relapses). Patients not successfully treated at the end of 12 weeks entered the Markov process in the "failure" state and remained there until they transitioned to the "success" or "death" states. Patients were transitioned from state to state on a weekly cycle based on probabilities derived from the GCA study. The mean survival times in this model were 174 weeks, 150.4 weeks, and 116.1 weeks for the voriconazole, itraconazole, and $\mathrm{CAB}$ groups respectively (see Table 4). ${ }^{24}$

Jansen and colleagues conducted a similar study for a German population. They used a decision tree and Markov model to simulate the 12 week and lifetime costs and outcomes of voriconazole and amphotericin B as first-line treatments for invasive aspergillosis. ${ }^{28}$ The mean survival times in this model were 174 weeks and 116.1 weeks for the voriconazole and $\mathrm{CAB}$ groups respectively (see Table 4). ${ }^{28}$ 


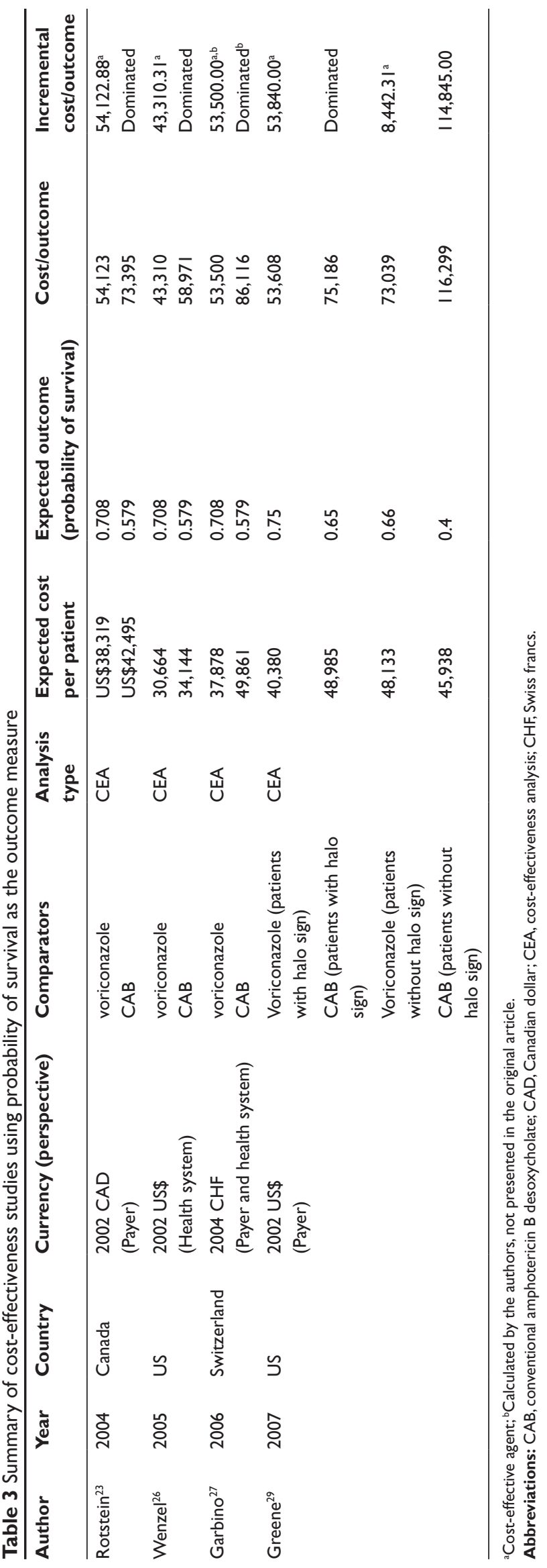

Ament and colleagues created a model to compare voriconazole and L-AMB as first-line therapy. After severe toxicity or treatment failure, a patient in the model could be switched to any combination of other antifungal agents. ${ }^{31}$ Seven different treatment combinations were compared in the final analysis: 1) voriconazole as first-line and L-AMB plus caspofungin as second-line therapy, 2) voriconazole as firstline and caspofungin as second-line therapy, 3 ) voriconazole as first-line and $\mathrm{CAB}$ as second-line therapy, 4) voriconazole as first-line and L-AMB as second-line therapy, 5) L-AMB as first-line and voriconazole plus caspofungin as secondline therapy, 6) L-AMB as first-line and voriconazole as second-line therapy, 7) L-AMB as first-line and caspofungin as second-line therapy. Probabilities used in the model were derived from multiple published studies. The mean survival times ranged from 1.109 to 1.307 years as summarized in Table $4 .{ }^{31}$

Four additional studies are described in greater detail to highlight their unique designs or contributions to the literature. Dominguez-Gil and colleagues conducted a cost minimization analysis comparing voriconazole and caspofungin from a hospital perspective. Based on probabilities obtained from multiple published studies, the authors determined or assumed that both agents would have equal efficacy. The average cost of the voriconazole-treated group was $€ 1,132$ lower than the caspofungin-treated group (see Table 5). ${ }^{32}$

Green and colleagues used a subset of patients from the GCA study that had thoracic computed tomographic (CT) scans at baseline to look at the cost-effectiveness of voriconazole compared to $\mathrm{CAB} .{ }^{29}$ They divided the patients into two groups, those with and without a halo sign on the CT scan at baseline. The probabilities of toxicity and success in these patients were used to model the expected cost and outcomes in each group. The probability of survival at 12 weeks was the outcome measure. Both groups had similar costs, but the group with the halo sign had better survival rates than the group without the sign. This resulted in lower costs per outcome in the group with the halo sign. Voriconazole was the most cost-effective agent in both groups (see Table 3). ${ }^{29}$

Wingard and colleagues conducted a cost analysis using data from the GCA study as the primary source of outcomes data. ${ }^{30}$ Consideration was not given to any outcome measure, but the patients were subdivided into survivors and nonsurvivors. The group treated with voriconazole had lower treatment costs compared to the group treated with $\mathrm{CAB}$ resulting in US\$16,758 savings in the survivor group. Conversely, the non-survivor group treated with $\mathrm{CAB}$ had savings of US\$10,176 compared to the voriconazole group. 


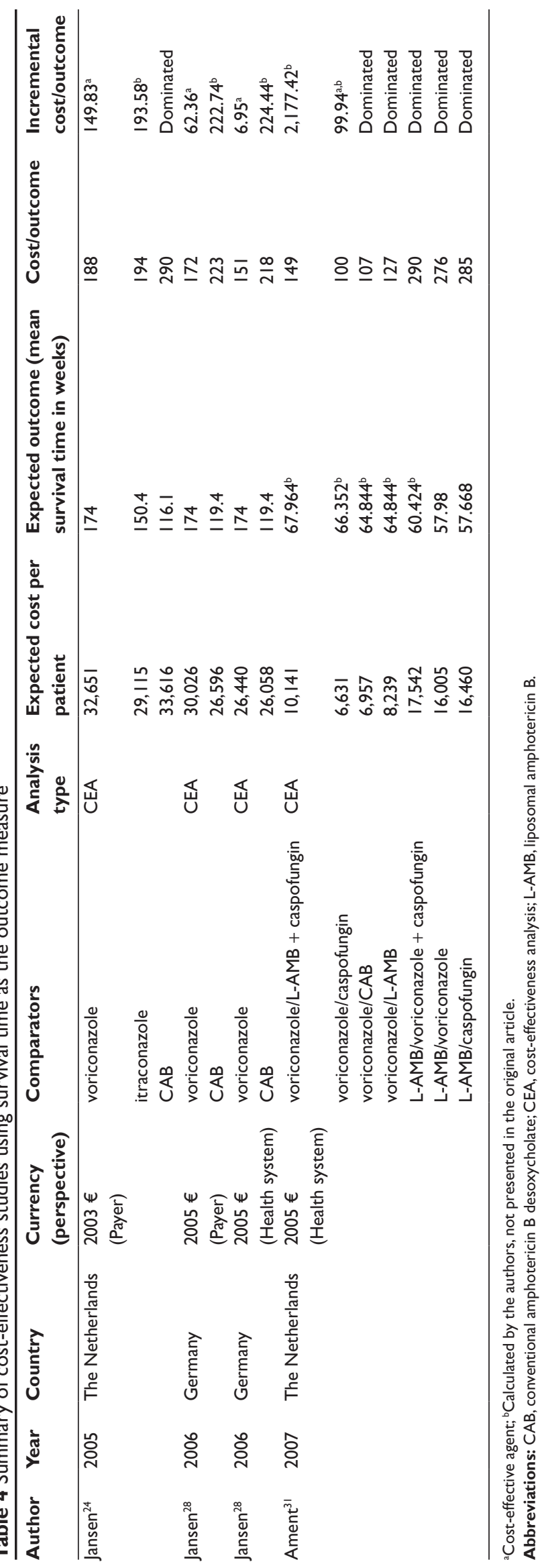

When all patients were considered together, patients treated with voriconazole had a US $\$ 4,997$ savings compared to patients treated with $\mathrm{CAB}$ (see Table 5). ${ }^{30}$

Overall the results of all of the analyses were robust to the assumptions made during the modeling process. This means that the results of the analysis did not change when the key assumptions of the models were tested in sensitivity analyses (ie, varied from high to low values). However the results of four studies were sensitive to drastic changes in either costs, the weight of the patient used to estimate the medication dose, or the renal toxicity of CAB. ${ }^{23,27,31,32}$ In the model used by Roststein and colleagues, patients were switched to L-AMB due to toxicity or treatment failure of voriconazole or CAB. If the cost of L-AMB was cut in half, then the $\mathrm{CAB}$ arm became more costeffective. ${ }^{23}$ In the model used by Garbino and colleagues, when the renal toxicity of amphotericin B was assumed to be less than $7 \%$, then the $\mathrm{CAB}$ arm became more costeffective. ${ }^{27}$ The results published by Dominguez-Gil and colleagues were robust as long as the patients weight was less than $102 \mathrm{~kg}^{32}$

Testing the conclusions of the pharmacoeconomic analyses with sensitivity analyses described above is one way to validate study results. In an attempt to further validate the results of a similar pharmacoeconomic analysis that has not been published, Van Campenhout and colleagues compared the results of the model to actual data collected from 116 patients in 13 different hematology-oncology intensive care settings in Belgium. ${ }^{34}$ The clinical response rate at 12 weeks was similar (53\% from the model, $50 \%$ from the observational study). However the model underestimated the mortality rates at 84 days ( $29 \%$ vs $42 \%$ ) and slightly overestimated the per-patient cost of hospitalizations (€21,298 vs $€ 19,674) .{ }^{34}$

Two of the studies included in this review accounted for the cost of the galactomannan assays, ${ }^{24,28}$ but none included the costs of voriconazole levels. The galactomannan assay is a diagnostic test, therefore the cost of this test would be incurred regardless of the therapy selected. In other words, including the cost of this diagnostic test in any of the pharmacoeconomic analyses would not change the results. The current treatment guidelines do not address the routine use of voriconazole levels. An observational study by Pascual and colleagues evaluated the impact of measuring voriconazole blood levels. ${ }^{6}$ Patient with troughs less than $1 \mathrm{mg} / \mathrm{L}$ had worse outcomes compared to those with troughs between 1 and $5.5 \mathrm{mg} / \mathrm{L}$, while patients with troughs higher than $5.5 \mathrm{mg} / \mathrm{L}$ had more toxicity. ${ }^{6}$ 
Table 5 Summary of cost minimization and cost analysis studies

\begin{tabular}{|c|c|c|c|c|c|c|c|c|}
\hline Author & Year & Country & Currency & Comparators & $\begin{array}{l}\text { Analysis } \\
\text { Type }\end{array}$ & $\begin{array}{l}\text { Expected cost } \\
\text { per patient }\end{array}$ & Cost difference & Comments \\
\hline \multirow[t]{2}{*}{ Dominguez-Gil $^{32}$} & 2007 & Spain & $2006 €$ & voriconazole & CMA & 6,042 & $-1,132.18$ & Cost-effective agent \\
\hline & & & & caspofungin & & 7,174 & & Dominated \\
\hline \multirow[t]{6}{*}{ Wingard ${ }^{30}$} & 2007 & US & 2005 US\$ & Voriconazole (all patients) & $\mathrm{CA}$ & 78,860 & $-4,997.00$ & Cost savings \\
\hline & & & & CAB (all patients) & & 83,857 & & \\
\hline & & & & Voriconazole (survivors) & & 79,913 & $-16,758.00$ & Cost savings \\
\hline & & & & $\mathrm{CAB}$ (survivors) & & 96,671 & & \\
\hline & & & & $\begin{array}{l}\text { Voriconazole } \\
\text { (non-survivors) }\end{array}$ & & 76,326 & $10,176.00$ & No cost savings \\
\hline & & & & $\mathrm{CAB}$ (non-survivors) & & 66,150 & & \\
\hline
\end{tabular}

Abbreviations: $C A B$, conventional amphotericin B desoxycholate; $C A$, cost analysis; $C M A$, cost-minimization analysis.

The median number of troughs drawn per patient was 4.5 with the first drawn a median of 5 days after therapy and every 7 days thereafter. The cost of this test is difficult to identify; however, the Centers for Medicare and Medicaid Services (CMS) 2009 Clinical Diagnostic Laboratory Fee Schedule limits for other drug assays ranges from US $\$ 17.40$ to US\$27.00. ${ }^{35}$ Thus if one assumes the cost to a payer would be the upper end of this limit (US\$27 per trough) with five troughs per patient, then an additional US\$135 would be added to the cost of therapy. When the cost of the assay is added to the expected costs calculated in each of the US-based pharmacoeconomic studies in this review, the results do not change. Voriconazole remains the most cost-effective therapy.

\section{Conclusions}

Amphotericin B and its lipid formulations, itraconazole, voriconazole and caspofungin, are all indicated for the primary treatment of invasive aspergillosis; however, voriconazole is preferred. ${ }^{1}$ The GCA study by Herbrecht and colleagues provided the clinical evidence to support voriconazole's clinical role. ${ }^{22}$ This publication also served as the basis for multiple pharmacoeconomic analyses which demonstrated that voriconazole is cost-effective in the primary treatment of invasive aspergillosis.

Of the agents indicated for the primary treatment of invasive aspergillosis, the US average wholesale price of L-AMB is the highest followed by caspofungin, voriconazole, itraconazole, and $\mathrm{CAB} .{ }^{16}$ While the acquisition costs of voriconazole are higher than those of conventional amphotericin B, the toxicity profile and treatment success rate associated with voriconazole result in lower total treatment costs per successfully treated patient. ${ }^{23-30}$

\section{Disclosure}

The authors declare no conflicts of interest.

\section{References}

1. Walsh TJ, Anaissie EJ, Denning DW, et al. Treatment of aspergillosis: clinical practice guidelines of the infectious diseases society of America. Clin Infect Dis. 2008;46(3):327-360.

2. Maschmeyer G, Haas A, Cornely OA. Invasive aspergillosis epidemiology, diagnosis and management in immunocompromised patients. Drugs. 2007;67(11):1567-1601.

3. Ruping MJ, Vehreschild JJ, Cornely OA. Patients at high risk of invasive fungal infections: when and how to treat. Drugs. 2008;68(14):1941-1962.

4. Segal BH. Aspergillosis. New Engl J Med. 2009;360(18):1870-1884.

5. Patterson TF. Aspergillus species. In: Mandell GL, Bennett JE, Dolin R, editors. Mandell, Douglas, and Bennett's principles and practice of infectious diseases. 6th ed. Philadelphia: Elsevier Inc; 2005. p. 2958-2972.

6. Pascual A, Calandra T, Bolay S, Buclin T, Bille J, Matchetti O. Voriconazole therapeutic drug monitoring in patients with invasive mycoses improves efficacy and safety outcomes. Clin Infect Dis. 2008;46(2):201-211.

7. Howard A, Hoffman J Sheth A. Clinical application of voriconazole concentrations in the treatment of invasive aspergillosis. Ann Pharmacother. 2008;42(12):1859-1864.

8. Dasbach EJ, Davies DM, Teutsch SM. Burden of aspergillosis-related hospitalizations in the United States. Clin Infect Dis. 2000;31(6): 1524-1528.

9. Wilson LS, Reyes CM, Stolpman M, Speckman J, Allen K, Beney J. The direct cost and incidence of systemic fungal infections. Value Health. 2002:5(1):26-34.

10. Databases, Tables and Calculators by Subject [CPI Inflation Calculator]. Washington: US Bureau of Labor Statistics. [updated 2009 May 7; cited 2009 May 7]. http://www.bls.gov/data/inflation_calculator.htm. Accessed 14 July, 2009.

11. Tong KB, Lau CJ, Murtagh K, Layton AJ, Seifeldin R. The economic impact of aspergillosis: analysis of hospital expenditures across patient subgroups. International J Infect Dis. 2009;13(1):24-36.

12. VFEND IV (voriconazole) for injection, VFEND tablets, VFEND for oral suspension [online-product labeling]. Pfizer. [revised 2008 March; cited 2009 May 8]. http://www.pfizer.com. Accessed 14 July, 2009.

13. Theuretzbacher U, Ihle F, Derendorf H. Pharmacokinetic/ pharmacodynamic profile of voriconazole. Clin Pharamacokinet. 2006;45(7):649-663.

14. Scott LJ, Simpson D. Voriconazole: a review of its use in the management of invasive fungal infections. Drugs. 2007;67(2):269-298. 
15. Zonios DI, Bennett JE. Update on azole antifungals. Semin Respir Crit Care Med. 2008;29(2):198-210.

16. Thompson Healthcare. Red Book. 2008 ed. Montvale, NJ: Thompson Healthcare; 2008.

17. Upton A, Kirby KA, Carpenter P, Boeckh M, Marr KA. Invasive aspergillosis following hematopoietic cell transplantation: outcomes and prognostic factors associated with mortality. Clin Infect Dis. 2007;44(4):531-540.

18. Cordonnier C, Ribaud P, Herbrecht R, et al. Prognostic factors for death due to invasive aspergillosis after hematopoietic stem cell transplantation: a 1-year retrospective study of consecutive patients at French transplantation centers. Clin Infect Dis. 2006;42(7):955-963.

19. Marr KA, Carter RA, Crippa F, Wald A, Corey L. Epidemiology and outcome of mould infections in hematopoietic stem cell transplant recipients. Clin Infect Dis. 2002;34(7):909-917.

20. Lin SJ, Schranz J, Teutsch SM. Aspergillosis case-fatality rate: systematic review of the literature. Clin Infect Dis. 2001;32(3): 358-366.

21. Neofytos D, Horn D, Anaissie E, et al. Epidemiology and outcome of invasive fungal infection in adult hematopoietic stem cell transplant recipients: analysis of multicenter prospective antifungal therapy (PATH) alliance registry. Clin Infect Dis. 2009;48(12):265-273.

22. Herbrecht R, Denning DW, Patterson TF, et al. Voriconazole versus amphotericin B for the primary therapy of invasive aspergillosis. N Engl $J$ Med. 2002;347(6):408-415.

23. Rotstein C, Laverdière M, Marciniak A, Ali F. An economic evaluation of voriconazole versus amphotericin B for the treatment of invasive aspergillosis in Canada. Can J Infect Dis Med Microbiol. 2004;15(5):277-284.

24. Jansen JP, Meis JF, Blijlevens NM, van't Wout JW. Economic evaluation of voriconazole in the treatment of invasive aspergillosis in the Netherlands. Curr Med Res Opin. 2005;21(10):1535-1546.

25. Lewis JS, Boucher HW, Lubowski TJ, Ambegaonkar AJ, Day DL, Patterson TE. Cost advantage of voriconazole over amphotericin B deoxycholate for primary treatment of invasive aspergillosis. Pharmacotherapy. 2005;25(6):839-846.

26. Wenzel R, Del Favero A, Kibbler C, et al. Economic evaluation of voriconazole compared with conventional amphotericin $\mathrm{B}$ for the primary treatment of aspergillosis in immunocompromised patients. $J$ Antimicrob Chemother. 2005;55(3):352-361.
27. Garbino J, Schnetzler G, Roberts C. Invasive aspergillosis: is treatment with "inexpensive" amphotericin B cost saving if "expensive" voriconazole is only used on demand? Swiss Med Wkly. 2006;136(39-40):624-630.

28. Jansen JP, Kern WV, Cornely OA, et al. Economic evaluation of voriconazole versus conventional amphotericin B in the treatment of invasive aspergillosis in Germany. Value Health. 2006;9(1):12-23.

29. Greene RE, Mauskopf J, Roberts CS, Zyczynski T, Schlamm HT. Comparative cost-effectiveness of voriconazole and amphotericin B in treatment of invasive pulmonary aspergillosis. Am J Health Syst Pharm. 2007;64(24):2561-2568.

30. Wingard JR, Herbrecht R, Mauskopf J, Schlamm HT, Marciniak A, Roberts CS. Resource use and cost of treatment with voriconazole or conventional amphotericin B for invasive aspergillosis. Transpl Infect Dis. 2007;9(3):182-188.

31. Ament AJHA, Hubben MWA, Verweij T, Poynton C, Kumar RN, Jansen JP. Economic evaluation of targeted treatments of invasive aspergillosis in adult haematopoietic stem cell transplant recipients in the Netherlands: a modeling approach. J Antimicrob Chemother. 2007;60(2):385-393.

32. Dominguez-Gil A, Martin I, Vargas MG, Del Castillo A, Diaz S, Sanchez C. Economic evaluation of voriconazole versus caspofungin for the treatment of invasive aspergillosis in Spain. Clin Drug Investig. 2007;27(3):197-205.

33. Drummond MF, O'Brien B, Stoddart GL, Torrance GW, editors. Methods for the Economic Evaluation of Health Care Programmes, 2nd ed. Oxford: Oxford University Press; 1997.

34. Van Campenhout H, Marbaix S, Derde MP, Annemans L. Voriconazole treatment of invasive aspergillosis: real-world versus health-economic model results. Clin Drug Investig. 2008;28(8):509-521.

35. Clinical Diagnostic Laboratory Fee Schedule [2009 Clinical Diagnostic Laboratory Fee Schedule]. Baltimore: Centers for Medicare and Medicaid Services. [updated 2009 January; Cited 2009 June 10]. http:// www.cms.hhs.gov/ClinicalLabFeeSched/02_clinlab.asp\#TopOfPage. Acessed 13 July, 2009.
ClinicoEconomics and Outcomes Research

\section{Publish your work in this journal}

ClinicoEconomics \& Outcomes Research is an international, peerreviewed open-access journal focusing on Health Technology Assessment, Pharmacoeconomics and Outcomes Research in the areas of diagnosis, medical devices, and clinical, surgical and pharmacological intervention. The economic impact of health policy and health systems

\section{Dovepress}

organization also constitute important areas of coverage. The manuscript management system is completely online and includes a very quick and fair peer-review system, which is all easy to use. Visit http://www.dovepress.com/testimonials.php to read real quotes from published authors. 